\title{
Dysfunction of the stress-responsive FOXC1 transcription factor contributes to the earlier-onset glaucoma observed in Axenfeld-Rieger syndrome patients
}

\author{
This article has been corrected since Online Publication and a corrigendum has also been published
}

\author{
YA Ito ${ }^{1}$, IS Goping ${ }^{2}$, F Berry ${ }^{1,3}$ and MA Walter ${ }^{*, 1,4}$
}

Mutations in the Forkhead Box C1 (FOXC1) transcription factor gene are associated with Axenfeld-Rieger syndrome (ARS), a developmental disorder affecting structures in the anterior segment of the eye. Approximately $75 \%$ of ARS patients with FOXC1 mutations develop earlier-onset glaucoma. Constant exposure of the trabecular meshwork (TM), located in the anterior segment of the eye, to oxidative stress is predicted to be a risk factor for developing glaucoma. Stress-induced death of TM cells results in dysfunction of the TM, leading to elevated intraocular pressure, which is a major risk factor for developing glaucoma. FOXC1 is predicted to maintain homeostasis in TM cells by regulating genes that are important for stress response. In this study, we show that a member of the heat-shock 70 family of proteins, HSPA6, is a target gene of FOXC1. HSPA6 protein, which is only induced under severe oxidative stress conditions, has a protective function in human trabecular meshwork (HTM) cells. We also show that FOXC1 is anti-apoptotic as knocking down FOXC1 significantly decreases HTM cell viability. In addition, we show that FOXC1 itself responds to stress as exposure of cells to $\mathrm{H}_{2} \mathrm{O}_{2}$-induced oxidative stress reduces FOXC1 levels and activity. Conditions that decrease FOXC1 function, such as exposure of cells to oxidative stress and FOXC1 ARS mutations, compromise the ability of TM cells to effectively respond to environmental stresses. Dysfunction of FOXC1 contributes to the death of TM cells, an important step in the development of glaucoma.

Cell Death and Disease (2014) 5, e1069; doi:10.1038/cddis.2014.8; published online 20 February 2014

Subject Category: Neuroscience

Axenfeld-Rieger syndrome (ARS) is an autosomal-dominant disorder in which structures in the anterior segment of the eye are improperly developed. A spectrum of anterior segment anomalies is observed in ARS patients including iris hypoplasia, polycoria, corectopia, posterior embryotoxon, and peripheral anterior synechiae. Systemic anomalies may include facial dysmorphisms, dental anomalies, and redundant preumbilical skin. ARS patients are at an increased risk to develop glaucoma, a progressively blinding condition that results from the death of retinal ganglion cells. Glaucoma is an age-related disease as the risk of developing glaucoma significantly increases after age 40 years. ${ }^{1,2}$ Patients with ARS typically have juvenile-onset or early adult-onset (before age 40 years) glaucoma. Histopathological examination of ARS eyes revealed malformations in the trabecular meshwork (TM) and Schlemm's canal, ${ }^{3}$ both of which are essential for the proper drainage of aqueous humor from the anterior chamber of the eye to occur. Inefficient drainage of the aqueous humor often results in elevated intraocular pressure (IOP), which is a major risk factor for the development of glaucoma.

Mutations in the Forkhead Box C1 (FOXC1) gene are associated with ARS and glaucoma. ${ }^{4-6}$ FOXC1 belongs to the Forkhead box (FOX) family of transcription factors, which share an evolutionarily conserved DNA-binding domain known as the Forkhead domain. The vast majority of FOXC1 missense mutations identified in ARS patients are located within the forkhead domain, ${ }^{7}$ highlighting the importance of an intact DNA-binding domain for FOXC1 to function during ocular development. Molecular dissection of FOXC1 mutations show that many FOXC1 mutant proteins have decreased capacity to localize to the nucleus and/or bind to DNA, both properties that are essential for FOXC1 to function as an effective transcription factor. ${ }^{8-10}$

In addition to FOXC1's role during ocular development, FOXC1 appears to have a protective role in the adult eye. ${ }^{11}$ FOXC1 is expressed in the adult eye including the TM. ${ }^{12}$

\footnotetext{
${ }^{1}$ Department of Medical Genetics, University of Alberta, Alberta T6G 2H7, Canada; ${ }^{2}$ Department of Biochemistry, University of Alberta, Alberta T6G 2H7, Canada; ${ }^{3}$ Department of Surgery, University of Alberta, Alberta T6G 2H7, Canada and ${ }^{4}$ Department of Ophthalmology, University of Alberta, Alberta T6G 2H7, Canada ${ }^{*}$ Corresponding author: MA Walter, Department of Medical Genetics, University of Alberta, 839 Medical Sciences Building, Alberta T6G 2H7, Canada. Tel: +780 492 9044; Fax: +780 492 1998; E-mail: mwalter@ualberta.ca

Keywords: FOXC1; HSPA6; glaucoma; axenfeld-rieger syndrome; trabecular meshwork; stress response

Abbreviations: ARS, Axenfeld-Rieger syndrome; BS, binding site; ChIP, chromatin immunoprecipitation; FOX, forkhead box; HSPA6, heat-shock protein A6; HSF1, heat-shock transcription factor 1; HTM, human trabecular meshwork; $\mathrm{H}_{2} \mathrm{O}_{2}$, hydrogen peroxide; IOP, intraocular pressure; PARP-1, poly (ADP-ribose) polymerase 1 ; TM, trabecular meshwork

Received 28.8.13; revised 19.12.13; accepted 03.1.14; Edited by A Verkhratsky
} 
Cells of the TM are constantly exposed to aqueous humor, which is a source of oxidative stress. ${ }^{13}$ The levels of hydrogen peroxide $\left(\mathrm{H}_{2} \mathrm{O}_{2}\right)$ in human aqueous humor is greatly variable and can reach levels as high as $300 \mu \mathrm{M} .{ }^{14}$ As true of most cells, the cells of the TM have cellular defense mechanisms including the antioxidant system, proteolytic system, and regulation of stress-responsive genes that enable the cell to quickly and efficiently adapt and survive in a dynamic environment. ${ }^{15-18}$ Compromised ability of the TM to respond to stress is predicted to result in TM cell death ${ }^{19-21}$ and subsequently in TM dysfunction, contributing to the development of glaucoma. FOXC1 maintains homeostasis in the adult TM by regulating anti-apoptotic genes such as FOXO1a. ${ }^{11}$ We hypothesize that ARS patients with FOXC1 mutations have a compromised ability to respond to stress, in part due to impaired regulation of downstream target genes that are involved in stress response. The compromised ability to respond to stress is predicted to contribute to the earlier-onset glaucoma observed in ARS patients. In the present study, we examined the involvement of the FOXC1 transcription factor in the stress response pathway by characterizing a novel potential target gene that belongs to the HSP70 family of proteins, Heat-shock protein A6 (HSPA6), also known as $\mathrm{HSP} \mathrm{B}^{\prime}$. Furthermore, we tested the effect of $\mathrm{H}_{2} \mathrm{O}_{2}$-induced oxidative stress on FOXC1 in human trabecular meshwork (HTM) cells.

\section{Results}

HSPA6 was examined as a potential target gene of FOXC1 in cultured HTM cells. This heat-shock protein was previously identified as a potential FOXC1 target gene by microarray analysis using a hormone-responsive FOXC1 construct. $^{11}$ Northern blot analysis and qPCR analysis both revealed that FOXC1 knockdown resulted in a significant decrease in HSPA6 RNA levels compared with control siRNA-transfected cells $(P$-value $=0.01$, Student's $t$-test; Figure 1$)$. FOXC1 knockdown was achieved by transfection with siRNA against FOXC1 and knockdown efficiency was confirmed by immunoblot analysis (Supplementary Figure 1).

To confirm that HSPA6 is a downstream target gene of the FOXC1 transcription factor, chromatin immunoprecipitation (ChIP) assay was used to identify FOXC1-binding sites (BSs) upstream of the HSPA6 transcription start site (Figure 2). Potential FOXC1 BSs in the intergenic region upstream of the HSPA6 gene were identified by using a FOXC1 DNA BS matrix and the Possum program. All identified potential FOXC1 BSs were examined by ChIP assay. ChIP assays revealed that endogenous FOXC1 binds to DNA within the BS1 region, located $\sim 4800 \mathrm{bp}$ upstream of the HSPA6 transcription start site and to a 300-bp DNA segment (BS3), containing two consensus FOX BSs located $\sim 1300 \mathrm{bp}$ upstream of the transcription start site (Figure 2). ChIP-PCR indicated that FOXC1 does not bind to the potential site within the $\mathrm{BS} 2$ region, however (Figure 2).

HSPA6 protein levels were examined to further validate HSPA6 as a downstream target of FOXC1. HSPA6 has been shown to have little or no basal protein expression and to be only induced upon exposure to extreme conditions of stress. ${ }^{22-25}$ Consistent with this idea, HSPA6 protein was not
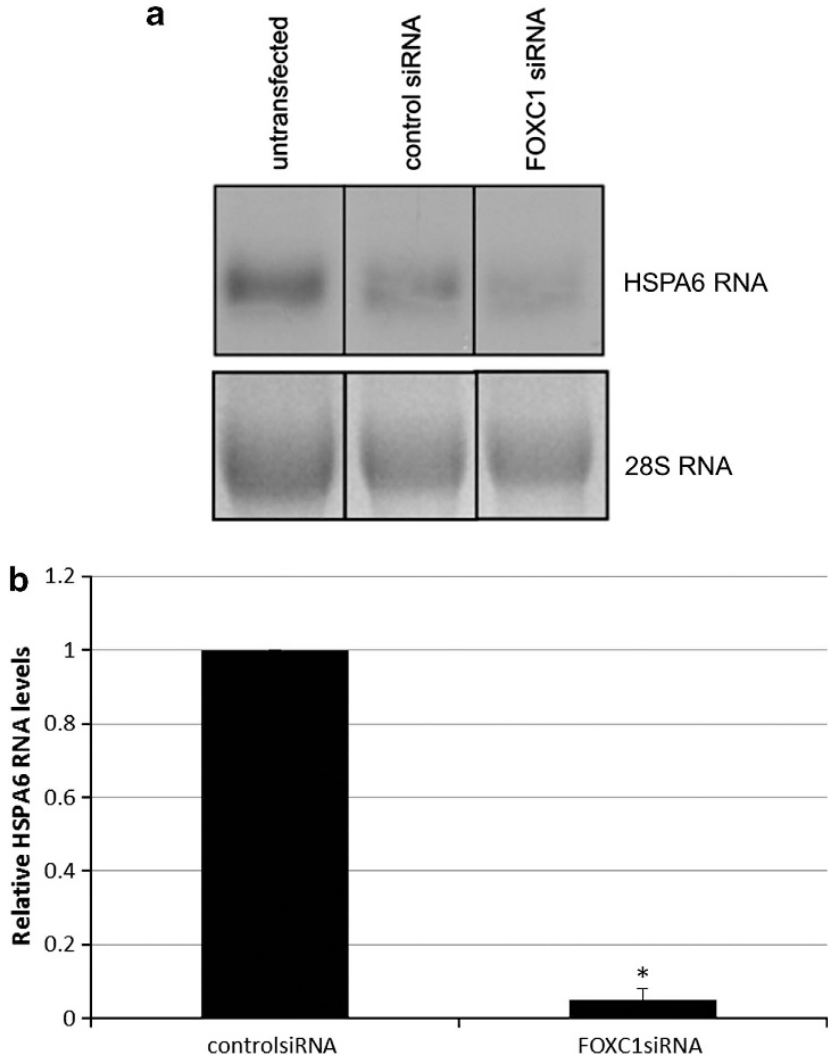

Figure 1 HSPA6 is a target gene of FOXC1 in HTM cells. HTM cells were transfected with FOXC1 siRNA or control siRNA. After transfection, RNA was harvested and subjected to (a) northern blot analysis or (b) GPCR analysis. (a) A HSPA6 probe was radiolabeled and hybridized to the blot. Relative to control siRNA, HSPA6 RNA levels significantly decreased when FOXC1 was knocked down $(P=0.01, n=4$, Student's $t$-test). (b) gPCR results show that relative to control siRNA, HSPA6 RNA levels significantly decreased after FOXC1 was knocked down $\left({ }^{\star} P<0.01, n=4\right.$, Student's $t$-test)

detected by immunoblot analysis under normal untreated conditions (Figure 3a). Thus, to examine HSPA6 protein, HTM cells were subjected to a two-step $\mathrm{H}_{2} \mathrm{O}_{2}$ treatment, where cells were first pre-conditioned with a lower dose of $\mathrm{H}_{2} \mathrm{O}_{2}$, and then treated with a second higher dose of $\mathrm{H}_{2} \mathrm{O}_{2}$ (Figure $3 \mathrm{~b}$ ). Neither single doses $(500 \mu \mathrm{M}=$ 'low dose' or $1000 \mu \mathrm{M}=$ 'high dose') of $\mathrm{H}_{2} \mathrm{O}_{2}$ resulted in HSPA6 protein detection. HSPA6 protein was only detected after two treatments of $\mathrm{H}_{2} \mathrm{O}_{2}$ (500 $\mu \mathrm{M}$ followed by $1000 \mu \mathrm{M}=$ 'severe dose'; Figure 3a). Thus, the two-step $\mathrm{H}_{2} \mathrm{O}_{2}$ treatment resulted in a severe oxidative stress condition, in which HSPA6 protein was induced. Under severe oxidative stress conditions, HSPA6 protein levels increased $76 \%$ when FOXC1 was knocked down relative to the control siRNA-transfected cells in which there were initially normal FOXC1 protein levels before treatment $(P=0.02$, Student's $t$-test; Figure $4 a)$. Similarly, analysis of HSPA6 RNA by qPCR indicate that under severe oxidative stress conditions, HSPA6 RNA levels increase when FOXC1 is knocked down (Figure 4b). Taken together, these experiments confirm that HSPA6 is a target gene of the FOXC1 transcription factor.

Members of the HSP70 family of proteins typically have a protective role in cells. ${ }^{25}$ To examine whether HSPA6 also has a protective role in HTM cells, HSPA6 was knocked down by 

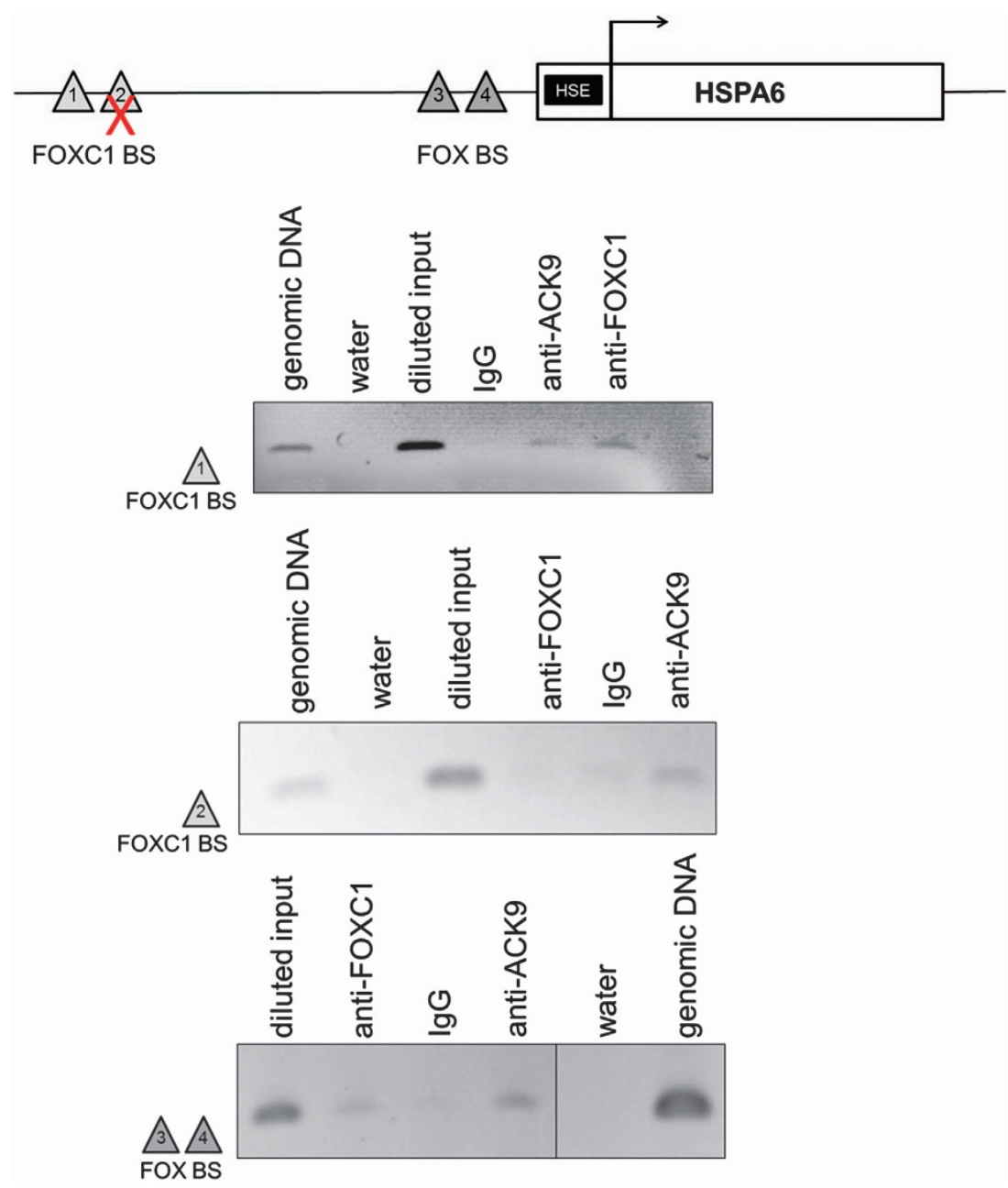

Figure 2 FOX-binding sites are present in the HSPA6 upstream region. Chromatin immunoprecipitation (ChIP) assay identified BS1, located $\sim 4800$ bp upstream of the HSPA6 transcription start site, as a bona fide FOXC1-binding site. The BS3 region containing two FOX consensus binding sites located $\sim 1300$ bp upstream of the HSPA6 transcription site was also able to bind FOXC1. HTM cells were crosslinked and immunoprecipitated against FOXC1. Acetylated histone 3 at lysine 9 (Ack9) and lgG were used as positive and negative controls for the ChIP technique, respectively. The immunoprecipitated DNA was amplified by PCR using primers flanking the potential FOXC1-binding site, identified by Possum software. Genomic DNA and water are positive and negative controls, respectively, for the PCR. Primer flanking BS2 did not amplify a product in ChIP products incubated with FOXC1 antibody, indicating that BS2 does not bind to FOXC1

transfection with siRNA against HSPA6 (Supplementary Figure 2). After exposure to a 'severe dose' of $\mathrm{H}_{2} \mathrm{O}_{2}$, which results in the induction of HSPA6 protein (Figure 3a), there were significantly more trypan blue-positive cells in HSPA6 siRNA-transfected cells $(64.7 \%)$ compared with control siRNA-transfected cells (58.7\%; $P$-value $=0.0027$, Student's $t$-test; Figure 5a). Examination of cleaved poly (ADP-ribose) polymerase 1 (PARP-1) protein levels by immunoblotting indicated that there was significantly more of this apoptotic marker when HSPA6 was knocked down in cells exposed to the 'severe dose' of $\mathrm{H}_{2} \mathrm{O}_{2}$ (Figure $5 b$ ). These results suggest that HSPA6 is an anti-apoptotic protein in severe stress conditions.

To further examine the potentially protective role of HSPA6, HSPA6 was overexpressed in HTM cells. Compared with empty vector-transfected cells, overexpression of HSPA6 resulted in a statistically significant $7.2 \%$ increase in cell viability after HTM cells were exposed to a 'high dose' of $\mathrm{H}_{2} \mathrm{O}_{2}(P$-value $=0.0028$, Student's $t$-test; Supplementary
Figure 3A). HSPA6 overexpression did not alter cell viability after HTM cells were exposed to the 'low dose' and 'severe dose' of $\mathrm{H}_{2} \mathrm{O}_{2}$. Interestingly, immunoblot analysis indicated that HSPA6 protein levels were lower in cells treated with 'low dose' and 'severe dose' treatments of $\mathrm{H}_{2} \mathrm{O}_{2}$ compared with cells that were untreated or treated with the 'high dose' of $\mathrm{H}_{2} \mathrm{O}_{2}$ (Supplementary Figure $3 \mathrm{~B}$ ). These results thus also suggest that HSPA6 protein has a protective role in severe stress conditions.

FOXC1 has previously been reported to promote resistance to oxidative stress. ${ }^{11}$ The protective role of FOXC1 was further examined in HTM cells that were subjected to various severities of oxidative stress. Under untreated conditions with normal FOXC1 levels, $78 \%$ of cells were viable (Figure $6 a$ ). However, there were significantly less viable cells $(69 \%)$ when FOXC1 was knocked under ' $n$ o $\mathrm{H}_{2} \mathrm{O}_{2}$ ' conditions ( $P$-value $<0.01$, Student's $t$-test). Also, relative to the control siRNA-transfected cells, there were significantly less viable cells in FOXC1 siRNA-transfected cells after exposure to 
a

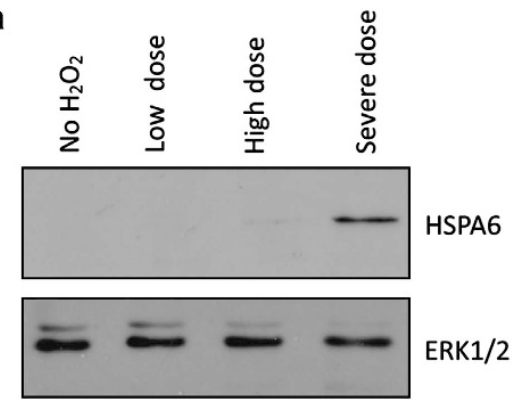

b $\mathrm{NoH}_{2} \mathrm{O}_{2}$

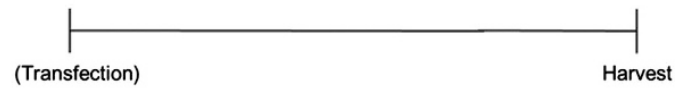

Low dose

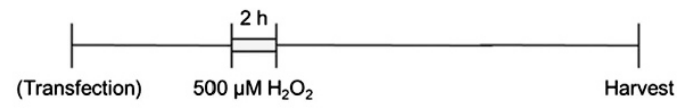

High dose
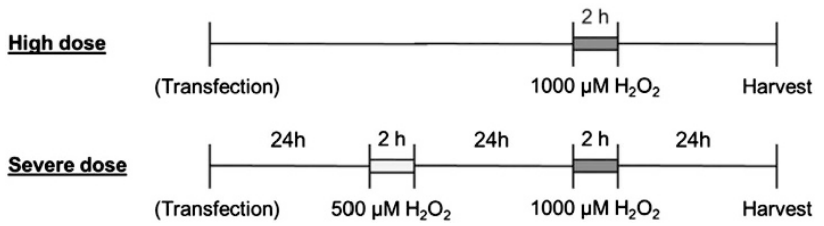

Figure 3 HSPA6 protein is present only after exposure to a severe dose of $\mathrm{H}_{2} \mathrm{O}_{2}$. (a) Protein lysates were resolved on a $10 \%$ SDS-PAGE and immunoblotted using antibodies against HSPA6 or ERK1/2. Immunoblot analysis shows the presence of HSPA6 protein only in HTM cells exposed to a severe dose of $\mathrm{H}_{2} \mathrm{O}_{2}$. (b) For overexpression or knockdown of FOXC1 or HSPA6, HTM cells were first transfected with either siRNA against the gene of interest or a plasmid construct, respectively. Approximately $24 \mathrm{~h}$ post transfection, HTM cells were exposed to a low dose $(500 \mu \mathrm{M})$, high dose $(1000 \mu \mathrm{M})$, or severe dose $(500 \mu \mathrm{M}$ followed by $1000 \mu \mathrm{M})$ of $\mathrm{H}_{2} \mathrm{O}_{2}$

either a 'low dose' or 'high dose' of $\mathrm{H}_{2} \mathrm{O}_{2}(P$-value $<0.01$, Student's $t$-test). As decreasing FOXC1 levels are associated with increased cell death, FOXC1 appears to have a role in preventing cell death under both normal and oxidative stress conditions. In cells transfected with control siRNA (normal FOXC1 levels), cleaved PARP-1 and cleaved caspase 7 protein levels both increased with the intensity of the $\mathrm{H}_{2} \mathrm{O}_{2}$ treatment (Figures $6 \mathrm{~b}$ and $\mathrm{c}$ ). When FOXC1 was knocked down, there was a significant increase in cleaved PARP-1 protein levels after exposure to both 'low dose' and 'high dose' oxidative stress conditions. Similarly, cleaved caspase 7 protein levels significantly increased when FOXC1 was knocked down in cells exposed to a 'high dose' and 'severe dose' of $\mathrm{H}_{2} \mathrm{O}_{2}$. Taken together, these results confirm that FOXC1 has a protective role by preventing apoptotic cell death.

The previous section shows that a reduction in FOXC1 levels, which inevitably results in a reduction of FOXC1 activity, is detrimental to cell viability. Many FOXC1 mutations found in ARS patients, including L130F, W152G, and S131L, have reduced FOXC1 activity. ${ }^{8-10}$ Thus, the consequence of these FOXC1 mutations on cell viability was examined. Overexpressing wild-type FOXC1 in HTM cells resulted in a significant $10.5 \%$ increase in cell viability compared with the empty vector-transfected cells when both types of transfected cells were treated with a 'high dose' of $\mathrm{H}_{2} \mathrm{O}_{2}(P$-value $=0.003$, Student's $t$-test; Figure $7 \mathrm{a})$. There was no difference in cell a
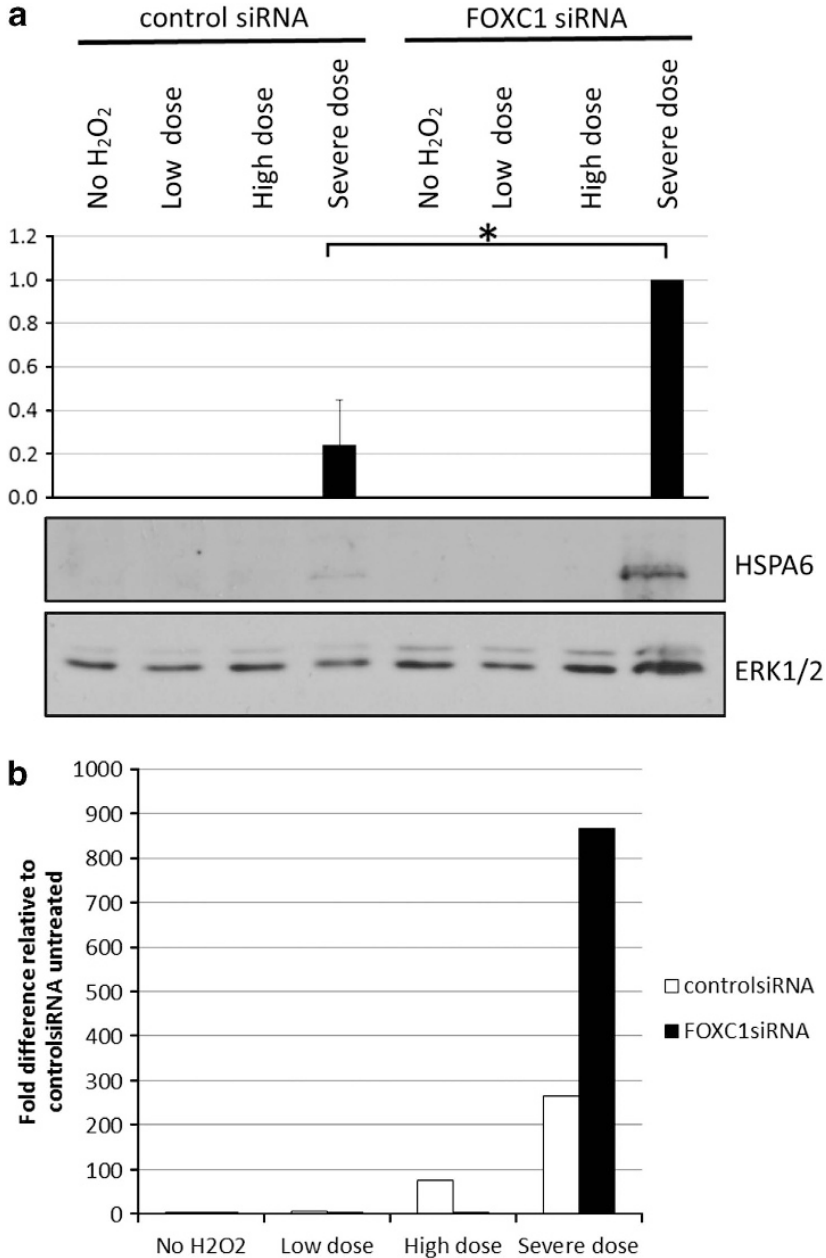

Figure 4 FOXC1 knockdown increases HSPA6 levels after exposure to a severe dose of $\mathrm{H}_{2} \mathrm{O}_{2}$. (a) Protein lysates were resolved on a $10 \%$ SDS-PAGE and immunoblotted using antibodies against HSPA6 or ERK1/2. Knocking down FOXC1 in severely oxidatively stressed cells resulted in a significant increase $\left(^{*}\right)$ in HSPA6 protein ( $P=0.02$. Student's $t$-test). (b) Total RNA was extracted and HSPA6 and HPRT1 RNA levels were quantified by GPCR. HSPA6 RNA levels were normalized to HPRT1 RNA levels. qPCR results show that HSPA6 RNA levels increase after exposure to a severe dose of $\mathrm{H}_{2} \mathrm{O}_{2}$, further confirming that HSPA6 is a target gene of FOXC1. Quantification is based on triplicated experiments

viability when FOXC1 was overexpressed in HTM cells when cells were untreated, or exposed to a 'low dose' or 'severe dose' of $\mathrm{H}_{2} \mathrm{O}_{2}$. In the absence of oxidative stress, cells overexpressing the L130F, W152G, or S131L FOXC1 mutation had significantly decreased cell viability compared with cells overexpressing wild-type FOXC1 $(P<0.01$ for all three mutations, Student's $t$-test) (Figure $7 \mathrm{~b}$ ). In addition, relative to wild-type FOXC1-transfected cells, there was increased cell death in HTM cells overexpressing the three FOXC1 mutants after treatment with either the 'low dose' or 'high dose' of $\mathrm{H}_{2} \mathrm{O}_{2}(P<0.01$ for all three mutations, Student's $t$-test). Thus, both siRNA knockdown and mutations of FOXC1 found in ARS patients have similar effects of reducing FOXC1 activity, and increasing cell death in response to stress.

Finally, immunoblot analysis showed a decrease in both wild-type and mutant exogenous FOXC1 protein levels after HTM cells were exposed to $\mathrm{H}_{2} \mathrm{O}_{2}$ (Figure 7c). In addition, 


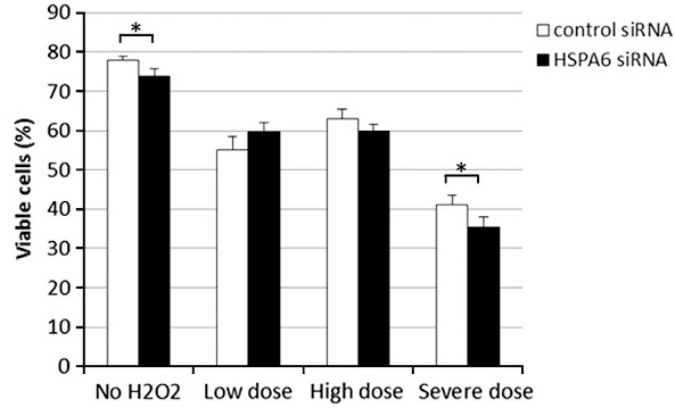

b
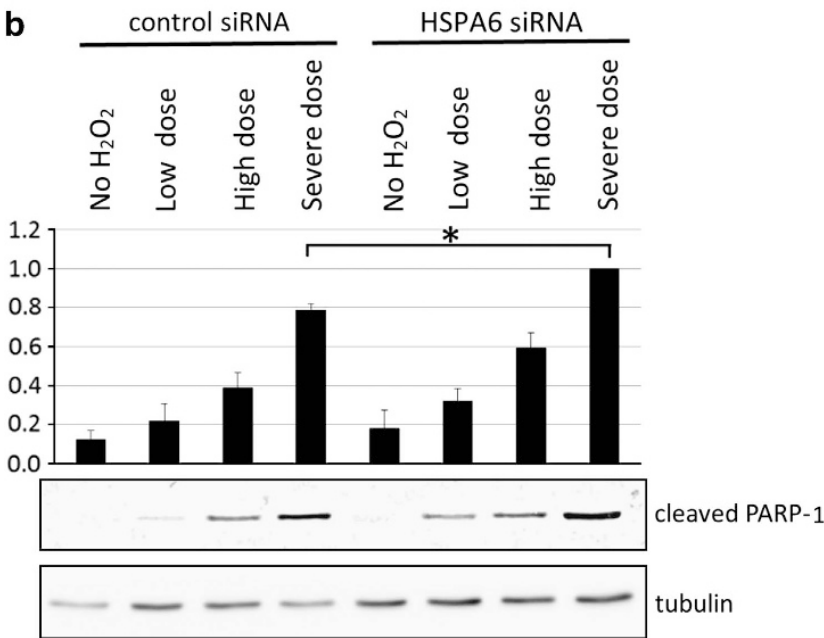

Figure 5 Knocking down HSPA6 increases apoptotic cell death after exposure of HTM cells to 'severe' $\mathrm{H}_{2} \mathrm{O}_{2}$-induced oxidative stress. (a) Cell viability was quantified by staining with trypan blue. HSPA6 knockdown resulted in a significant decrease $\left(^{*}\right)$ in cell viability in cells exposed to a 'severe dose' of $\mathrm{H}_{2} \mathrm{O}_{2}$. (b) Immunoblot analysis showed that decreasing HSPA6 significantly increased $\left(^{*}\right)$ cleaved PARP-1 levels after cells were exposed to a 'severe dose' of $\mathrm{H}_{2} \mathrm{O}_{2}$. Quantification is based on triplicated experiments

endogenous FOXC1 protein levels decreased after HTM cells were subjected to $\mathrm{H}_{2} \mathrm{O}_{2}$-induced oxidative stress (Figure 8a). FOXC1 protein levels did not recover even after $48 \mathrm{~h}$ post-treatment, as observed with the 'low dose' samples. The decrease in FOXC1 protein levels is not dependent on the severity of the $\mathrm{H}_{2} \mathrm{O}_{2}$ treatment as all three examined treatment conditions resulted in lower FOXC1 protein levels. Similarly, qPCR results showed that relative to untreated HTM samples, all three examined $\mathrm{H}_{2} \mathrm{O}_{2}$ treatment conditions resulted in a significant decrease in FOXC1 RNA levels $(P$-value $<0.01$, Student's $t$-test; Figure $8 \mathrm{~b}$ ). These experiments indicate that FOXC1 mRNA and protein levels decrease after exposure of cells to oxidative stress, and suggest that FOXC1 itself is a stress-responsive transcription factor. To test this idea, the ability of FOXC1 to transactivate a luciferase reporter gene with a synthetic FOXC1 BS was examined in HeLa cells. FOXC1 was able to transactivate the luciferase reporter gene under ' $n o \mathrm{H}_{2} \mathrm{O}_{2}$ ' conditions (Figure 8c). However, relative to no $\mathrm{H}_{2} \mathrm{O}_{2}$ treatment, transactivation levels were significantly reduced after exposure of cells to either a 'low dose' or 'severe dose' of $\mathrm{H}_{2} \mathrm{O}_{2}$-induced oxidative stress (Figure 8d). Interestingly, despite reduced FOXC1 protein levels, FOXC1

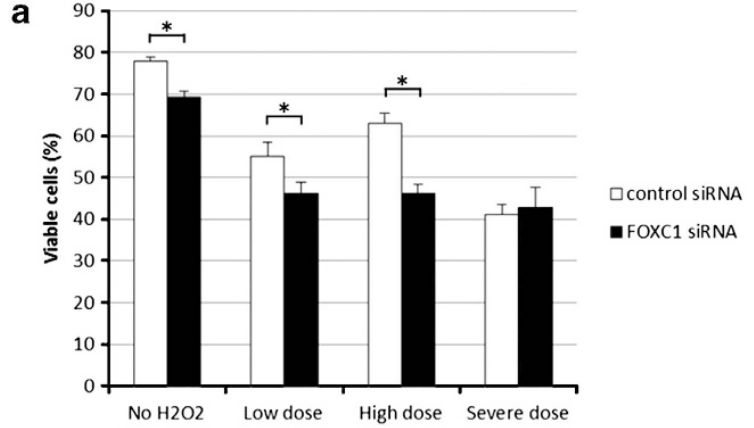

b
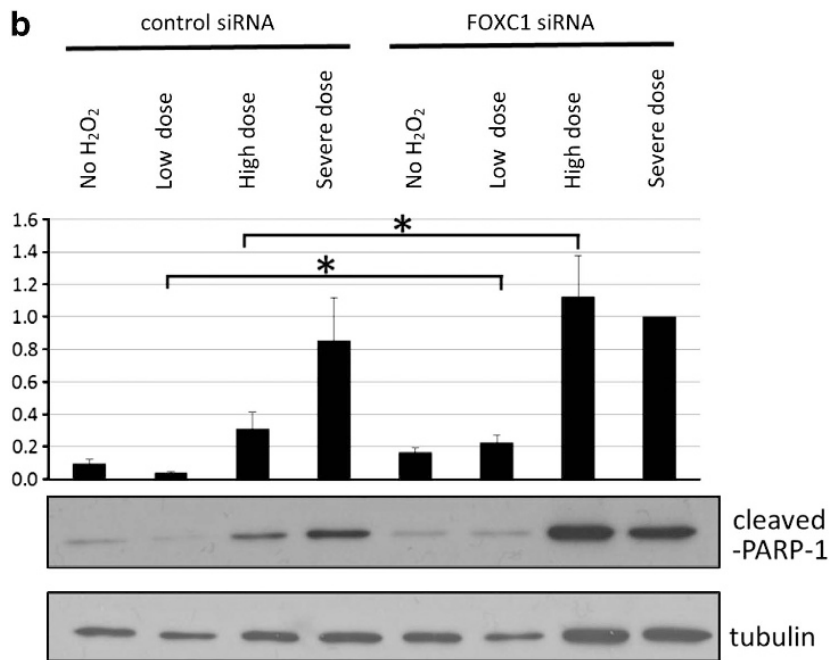

C

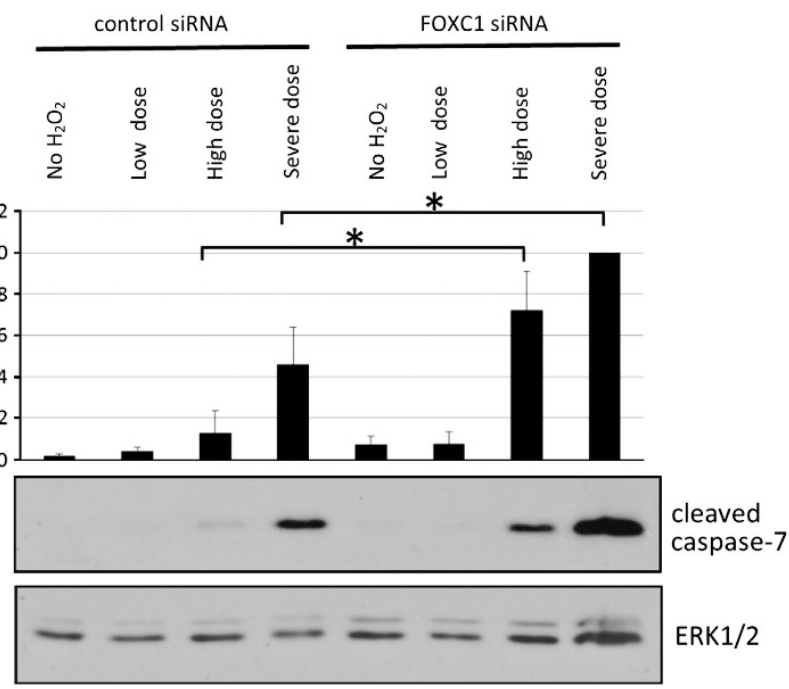

Figure 6 Knocking down FOXC1 increases apoptotic cell death after exposure of HTM cells to 'low dose' and 'high dose' $\mathrm{H}_{2} \mathrm{O}_{2}$-induced oxidative stress. (a) Cell viability was quantified by staining with trypan blue. Knocking down FOXC1 significantly decreased (*) cell viability in cells exposed to a 'low dose', 'high dose', and 'severe dose' of $\mathrm{H}_{2} \mathrm{O}_{2}$. Immunoblot analysis showed that $\left({ }^{*}\right)$ decreasing FOXC1 significantly increased the apoptotic markers (b) cleaved PARP-1 and (c) cleaved caspase-7 after cells were exposed to a 'low dose' and/or 'high dose' of $\mathrm{H}_{2} \mathrm{O}_{2}$. FOXC1 knockdown had no effect on cell viability or the apoptotic markers when cells were exposed to a 'severe dose' of $\mathrm{H}_{2} \mathrm{O}_{2}$. Quantification is based on triplicated experiments 
a

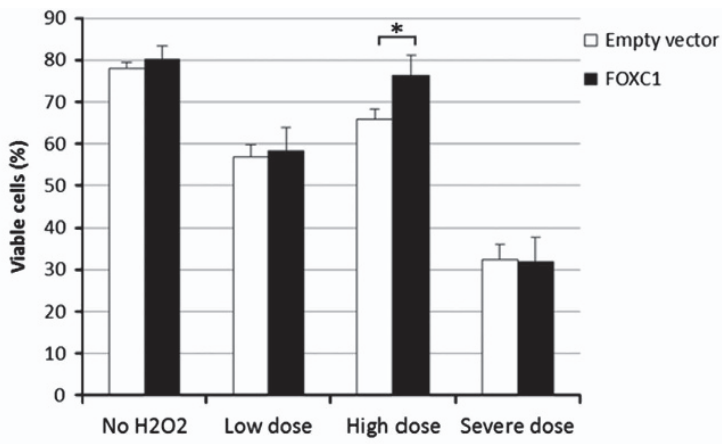

b

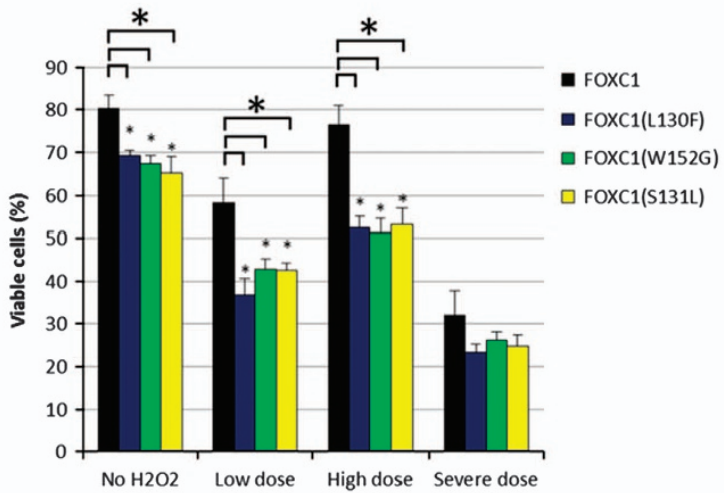

c

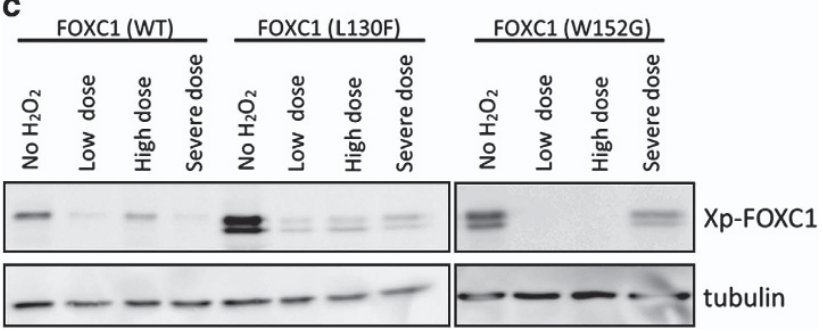

Figure 7 Overexpression of wild-type and mutant FOXC1s has differential effects on cell viability. HTM cells were transfected with (a) wild-type FOXC1 or (b) three mutant FOXC1s; L130F, W152G, and S131L. Transfected HTM cells were subjected to a low dose $(500 \mu \mathrm{M})$, high dose $(1000 \mu \mathrm{M})$, or severe dose $(500 \mu \mathrm{M}$ followed by $1000 \mu \mathrm{M}$ ) of $\mathrm{H}_{2} \mathrm{O}_{2}$. Cell viability was quantified by staining with trypan blue. Wild-type FOXC1 overexpression significantly increased (*) viability in cells exposed to a 'high dose' of $\mathrm{H}_{2} \mathrm{O}_{2}(P<0.01$, Student's $t$-test $)$. Under severe dose conditions, there was no significant difference between wild-type FOXC1 and the L130F, W152G, and S131L mutants with P-values of $0.06,0.20$, and 0.13 respectively. Mutant FOXC1 overexpression significantly decreased (*) viability in cells $(P<0.01$, Student's $t$-test). (c) Immunoblot analysis showed that FOXC1 protein levels decreased when HTM cells were exposed to $\mathrm{H}_{2} \mathrm{O}_{2}$-induced oxidative stress. Quantification is based on triplicated experiments

was able to transactivate the luciferase reporter gene after exposure to a 'high dose' of $\mathrm{H}_{2} \mathrm{O}_{2}$. Thus, FOXC1 has the greatest protective effect after exposure to a 'high dose' of $\mathrm{H}_{2} \mathrm{O}_{2}$.

\section{Discussion}

Because of the critical connection between cellular stress and the development of glaucoma, stress-induced genes are particularly interesting to study to better understand the role of stress response in the pathology of glaucoma. Our results indicated one such gene, HSPA6 as a target gene of the FOXC1 transcription factor, mutations of which cause ARS and earlier-onset glaucoma. HSPA6 homologs have been identified only in a subset of mammals. ${ }^{24}$ As no HSPA6 homologs have been identified in conventional model organisms such as rodents and fish, HTM cells were used here to examine HSPA6 function. HSP70 proteins typically have a protective role in cells through molecular chaperoning activities or interaction with various components of the apoptotic pathway such as Apaf-1 and AIF. ${ }^{26-33}$ Although the specific mechanisms of action have not been defined for HSPA6, similar mechanisms likely contribute to the protective ability of HSPA6 in HTM cells (Figure 5 and Supplementary Figure 3). HSPA6 is necessary for human colon cells to acquire cytoprotection in response to heat shock. ${ }^{24}$ However, under the experimental conditions in the present study, HTM cells did not acquire cytoprotection after preconditioning with a non-lethal dose of $\mathrm{H}_{2} \mathrm{O}_{2}$. Despite this difference, HSPA6 appears to nevertheless have a protective effect on both human colon and ocular cells. Finally, many heat-shock proteins including HSP70s function as 'danger signals' that are released from dying cells. ${ }^{33-35}$ In addition to the protective role of HSPA6 within cells, high levels of HSPA6 protein could potentially serve as a 'danger signal' in response to severe oxidative stress. ${ }^{24}$

Cellular stress has a complex effect upon the regulation of HSPA6 by FOXC1. Under normal non-stress conditions, FOXC1 binds to elements within the upstream regions of HSPA6 and significantly increases HSPA6 RNA expression (Figures 1 and 2). FOXC1 knockdown results in decreased HSPA6 RNA levels ${ }^{11}$ (Figure 1). However, under normal nonstress conditions, this HSPA6 RNA is not translated into protein as HSPA6 protein is only detected after exposure of cells to severe stress conditions (Figures 1 and 3 ). The positive correlation between HSPA6 transcript and protein levels in response to severe oxidative stress suggests that a transcriptional threshold must be reached for HSPA6 to be translated (Figure 4). Stress-induced changes to FOXC1 levels and activity might underlie the observation of increased HSPA6 protein levels with decreased FOXC1 levels. Exposure to oxidative stress itself alters FOXC1 transcript and protein levels, as well as activity (Figures 7 and 8). As both endogenous and exogenous FOXC1 protein levels decrease in response to oxidative stress, oxidative stress could affect the stability of FOXC1 protein. ${ }^{36}$ Although oxidative stress does not alter nuclear localization of FOXC1 protein (data not shown), other factors such as protein stability could be affected through post-translational modifications. Phosphorylation of the FOXC1 serine 272 residue has been shown to promote FOXC1 protein stability. ${ }^{37}$ Impairing phosphorylation of the serine 272 residue by substitution with an alanine residue (S272A) resulted in a less stable, but more transcriptionally active protein. ${ }^{37}$ The increased transactivation ability of FOXC1 despite the significant reduction in FOXC1 protein levels after exposure of HTM cells to a 'high dose' of $\mathrm{H}_{2} \mathrm{O}_{2}$ (Figure 8) could be due to a less stable, but more active FOXC1 protein. Furthermore, under severe stress conditions, wild-type FOXC1 does not have a protective advantage over the three mutant FOXC1 proteins examined (Figure 7). Thus, the initial low-dose treatment of $\mathrm{H}_{2} \mathrm{O}_{2}$ appears to disrupt the 
a

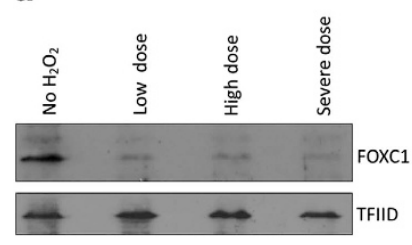

C

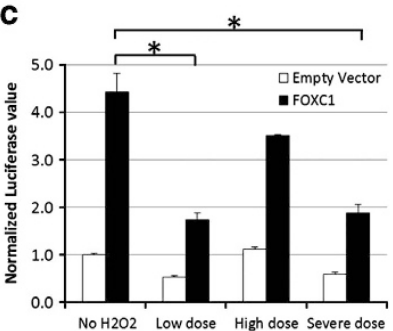

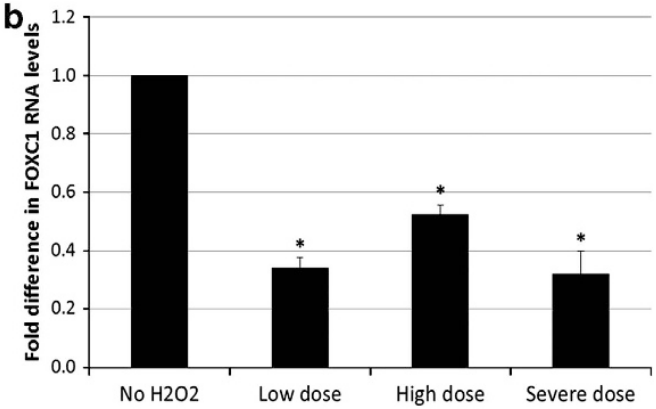

d

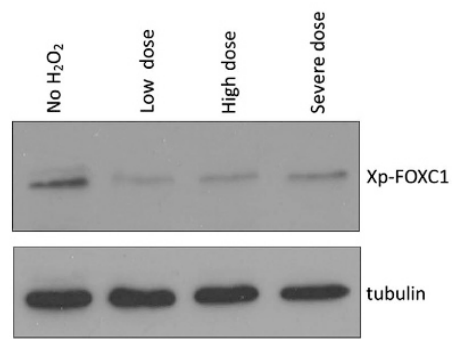

Figure $8 \quad \mathrm{H}_{2} \mathrm{O}_{2}$-induced oxidative stress decreases FOXC1 levels. (a) Nuclear extracts were resolved by $10 \%$ SDS-PAGE and immunoblotted using antibodies against endogenous FOXC1 and TFIID. (b) Total RNA was extracted and FOXC1 and HPRT1 RNA levels were quantified by qPCR. FOXC1 RNA levels were normalized to HPRT1 RNA levels. The * indicates a $P$ value $<0.01$ (Student's $t$-test) relative to 'no $\mathrm{H}_{2} \mathrm{O}_{2}$ ' samples. (c) $\mathrm{FOXC1}$ was able to transactivate the luciferase reporter gene under 'no $\mathrm{H}_{2} \mathrm{O}_{2}$ ' conditions. The transactivation ability of FOXC1 significantly decreased (*) in HeLa cells treated with a 'low dose' and 'severe dose' of $\mathrm{H}_{2} \mathrm{O}_{2}$. (d) Immunoblot analysis showed that exogenous FOXC1 protein levels decreased when cells were treated with $\mathrm{H}_{2} \mathrm{O}_{2}$. Lysates were resolved by $10 \%$ SDS-PAGE and immunoblotted using Xpress and tubulin antibodies. Quantification is based on triplicated experiments

functioning capacity of wild-type FOXC1 potentially through changes to its epigenetic landscape. Such stress-induced changes to FOXC1 would be predicted to alter the regulation of downstream target genes of FOXC1, including HSPA6 and should be further examined. Although HSPA6 was identified as a target of FOXC1 by a microarray method using a caged FOXC1 molecule designed to identify direct FOXC1 targets and contains upstream DNA elements that are bound by endogenous FOXC1 in ChIP assays, we cannot rule out the possibility that unknown factors intervene between FOXC1 and the HSPA6 regulatory region, especially under conditions of stress. In any case, the complex relation between FOXC1, HSPA6, and stress has an important role for the promotion of cell survival. Given the protective functions of HSPA6, dysregulation of HSPA6 in ARS patients with FOXC1 mutations could have a significant role in ARS-associated earlier-onset glaucoma.

FOXC1 is an anti-apoptotic protein ${ }^{11}$ as decreasing FOXC1 levels result in increased cell death under oxidative stress conditions (Figure 6). Thus, maintenance of FOXC1 activity appears to be essential for TM cell viability. In the present study, we show that oxidative stress conditions and FOXC1 mutations both compromise TM cell viability. Previous molecular characterization of FOXC1 mutants have revealed that the overall consequence of FOXC1 mutations found in ARS patients, including L130F and W152G, is decreased capacity to activate downstream target genes. ${ }^{9,10}$ In addition to downstream target genes that function during the development of anterior segment structures, FOXC1 mutations also disrupt the regulation of downstream target genes involved in executing a rapid and effective stress response during and after development. Our cell viability experiments show that even in the absence of stress, overexpression of mutant FOXC1 proteins result in a significant decrease in cell viability (Figure 7). Previous studies indicate that the L130F and W152G mutant proteins are misfolded, likely contributing to the formation of aggregates in the cytoplasm. ${ }^{9,10}$ As misfolded proteins inevitably place additional strain on the proteolytic system, these FOXC1 mutant proteins themselves could contribute to proteolytic stress even in the absence of environmental stresses.

Several factors are predicted to contribute to the earlieronset glaucoma observed in ARS patients with FOXC1 mutations. First, expression of FOXC1 mutants during development could result in malformation of tissues in the anterior segment of the eye, including the TM. Structural maldevelopment of the TM could result in inadequate aqueous humor drainage from the eye, leading to increased $\mathrm{IOP}$, which is a major risk factor for developing glaucoma. In addition to the role of FOXC1 during development, FOXC1 is predicted to function in maintaining homeostasis in the adult eye by mediating the stress response pathway. ${ }^{11}$ Under normal conditions, the FOXC1 transcription factor regulates a network of genes that involve both survival genes and apoptotic genes. The balanced regulation of these target genes by FOXC1 is likely essential to achieve homeostasis within a system. When HTM cells are exposed to environmental stresses, the decrease in FOXC1 levels appears to result in initiation of a different signaling program. A decrease in FOXC1 results in repression of anti-apoptotic genes, including FOXO1a, ${ }^{11}$ and could also induce pro-apoptotic genes that will result in apoptotic cell death. Exposure of HTM cells to severe environmental stresses stimulates a different subset of FOXC1 downstream target genes, including the 
anti-apoptotic HSPA6 gene (Supplementary Figure 4). In ARS patients with FOXC1 mutations, the TM cells are predicted to be more sensitive to environmental stresses because of both dysregulation of stress-responsive target genes such as HSPA6 and FOXO1a, ${ }^{11}$ and the additional strain put on the proteolytic system because of the presence of misfolded mutant FOXC1 protein. Also, in ARS patients, the copy of FOXC1 from the unaffected allele is predicted to be unable to compensate for the mutant allele during the stress response because exposure to oxidative stress reduces FOXC1 levels. Fluctuation of FOXC1 levels in the adult eye in response to stress may be part of the normal physiology of cells in the TM. However, in cells that are already compromised because of the expression of a FOXC1 mutation, fluctuations in wild-type FOXC1 levels in response to stress may further compromise the ability of TM cells to respond and adapt to stress, disrupting the overall functioning of the TM. Dysfunction of the TM likely contributes to the earlier-onset glaucoma observed in ARS patients with FOXC1 mutations.

As increasing the levels of FOXC1 promotes TM cell survival (Figure 7a), increasing FOXC1 levels may be an effective strategy for developing therapies for ARS patients. However, increased FOXC1 levels and/or overactivation of FOXC1 have also been associated with disease phenotypes. ${ }^{38-41} \mathrm{~A}$ balance in FOXC1 activity is necessary for proper development of the eye to occur. Both loss-of-function FOXC1 mutations and segmental duplications of the $6 \mathrm{p} 25$ region (resulting in additional copies of FOXC1) result in anterior segment dysgenesis. ${ }^{38-40,42}$ Another concerning factor is in human basal-like breast cancer, where high levels of FOXC1 are correlated with poor prognosis because of a more aggressive cellular phenotype that includes increased cell proliferation, migration, and invasion. ${ }^{41}$ These studies show that FOXC1 levels within a cell must be strictly regulated. The requirements of strict regulation of FOXC1 levels for normal development and cell phenotypes therefore suggests that FOXC1 is likely a poor target for direct therapeutic interventions for earlier-onset glaucoma. However, HSPA6 and other downstream targets of FOXC1 are intriguing potential therapeutic targets for preventing or delaying TM cell death.

\begin{abstract}
Materials and Methods
Mammalian cell culture and transfection. HTM cells were grown in Dulbecco's modified Eagle's medium (Life Technologies Inc., Burlington, ON, Canada), supplemented with $10 \%$ fetal bovine serum. Plasmid transfections were performed using a TransIT-LT1 (Mirus, Bio LLC, Madison, WI, USA) transfection reagent to DNA ratio of $5: 1$, according to the manufacturer's protocol. siRNA transfections were performed using Lipofectamine 2000 (Life Technologies), as previously described.11
\end{abstract}

Plasmids. The FOXC1 wild type, L130F, W152G, and S131L mutant constructs were previously assembled. ${ }^{8-10}$ The HSPA6 full-length CDNA (DNASU Plasmid Repository, Tempe, AZ, USA) was subcloned into the pcDNA3.1/nV5DEST vector (Life Technologies) in-frame to the V5 epitope by gateway technology. All vectors were sequenced to confirm that no nucleotide changes were introduced into the cDNA and that the cDNAs were in-frame to the epitopes.

Two-step $\mathrm{H}_{2} \mathrm{O}_{2}$ treatment. Twenty-four hours post transfection, cells were treated with $500 \mu \mathrm{M} \mathrm{H} \mathrm{O}_{2}$ for $2 \mathrm{~h}$, then allowed to recover in fresh media. Approximately $22 \mathrm{~h}$ post recovery, the cells were treated with $1000 \mu \mathrm{M} \mathrm{H}_{2} \mathrm{O}_{2}$ for $2 \mathrm{~h}$, then allowed to recover in fresh media for approximately $22 \mathrm{~h}$ before analysis (Figure 3b).
Heat-shock treatment. Approximately $48 \mathrm{~h}$ post transfection, HTM cells were heat shocked at $44^{\circ} \mathrm{C}$ for $2 \mathrm{~h}$. Cells were allowed to recover in fresh media pre-warmed to $37^{\circ} \mathrm{C}$ for $\sim 22 \mathrm{~h}$ before analysis.

Trypan blue staining. Twenty-four hours post transfection, HTM cells were exposed to the two-step $\mathrm{H}_{2} \mathrm{O}_{2}$ treatment before harvesting. Cells were trypsinized, then resuspended in $750 \mu \mathrm{l}$ PBS. Cell viability was analyzed by mixing equal volumes of resuspended cells with $0.4 \%$ trypan blue (Life Technologies Inc.). After addition of trypan blue, the cells were immediately analyzed by loading onto a hemocytometer. For each sample, cells in eight $1 \mathrm{~mm}^{2}$ squares were counted.

Protein analysis. For immunoblot analysis of endogenous FOXC1 protein, cells were harvested by scraping, lysed using Levin lysis buffer, ${ }^{43}$ and sonicated. For immunoblot analysis of all other proteins, cells were harvested by scraping, lysed using nuclear lysis buffer $(20 \mathrm{mM}$ HEPES $(\mathrm{pH} 7.6), 500 \mathrm{mM}$ $\mathrm{NaCl}, 1.5 \mathrm{mM} \mathrm{MgCl} 2,0.1 \%$ Triton-X 100, 20\% glycerol, $1 \mathrm{mM}$ dithiothreitol, $1 \mathrm{mM}$ phenylmethylsulfonylfluoride, $0.5 \%$ protease inhibitor cocktail (Sigma-Aldrich Canada Co., Oakville, ON, Canada)), and sonicated. Immunoblot analysis was carried out as previously described. ${ }^{44}$

Realtime qPCR. The QuantiTect SYBR Green PCR assay (QIAGEN, Toronto, ON, Canada) was used to quantitate FOXC1 or HSPA6 levels from reverse transcribed total RNA isolated from HTM cells as previously described. ${ }^{45}$ The following primers were used to detect FOXC1 RNA levels: forward 5'-CGGGTTGGAAAGGGATATTTA- $3^{\prime}$ and reverse $5^{\prime}$-CAAAATGTTCTGCTC CTCTCG-3'. The following primers were used to detect HSPA6 RNA levels: forward $5^{\prime}$-CAAGGTGCGCGTATGCTAC- $3^{\prime}$ and reverse $5^{\prime}$-GCTCATTGATGATCCG CAACAC- $3^{\prime}$.

Dual luciferase assay. The pGL3-TK reporter vector with the synthetic FOXC1 BSs was used for the transactivation assays. Twenty-four hours post transfection, HeLa cells were subjected to the two-step $\mathrm{H}_{2} \mathrm{O}_{2}$ treatment. Twentytwo hours post recovery, the luciferase assays were carried out using the dual luciferase assay kit as previously described. ${ }^{37}$ Each experiment was done in triplicate and was performed three times.

Northern blot analysis. Northern blot analysis was carried out as previously described. ${ }^{8}$ Twenty to thirty microgram of RNA was analyzed by northern blot on a formaldehyde agarose gel. A HSPA6 DNA fragment, unique to the $5^{\prime}$ untranslated region of HSPA6, was PCR amplified using the following primers: forward $5^{\prime}$-CAAGGTGCGCGTATGCTAC- $3^{\prime}$ and reverse $5^{\prime}$-GCTCATTGATGATCCGCA ACAC- $3^{\prime}$. HSPA6 RNA levels were normalized to $28 \mathrm{~S}$ levels and quantified using ImageJ. The level of $28 \mathrm{~S}$ ribosomal RNA was determined by visualization with the addition of ethidium bromide to the gel.

ChIP analysis. ChIP analysis was carried out as previously described. ${ }^{46}$ Protein A/G PLUS-Agarose beads (Santa Cruz Biotechnology, Dallas, TX, USA) were used in the ChIP experiments. Lysates were incubated with $4 \mu \mathrm{g}$ of FOXC1 antibody (OriGene Technologies, Inc., Rockville, MD, USA), $4 \mu \mathrm{g}$ of Histone H3 acetylated at lysine 9 antibody (Cell Signaling Technology, Inc., Danvers, MA, USA), or rabbit IgG (Cell Signaling Technology, Inc.) overnight before addition of protein beads. For each ChIP-PCR reaction, $1-5 \mu$ l of the purified DNA (i.e., ChIP reaction) was used as template. The primer sequences that were used for the ChIP-PCR are summarized in Supplementary Table 1 in the Supplementary Data.

\section{Conflict of Interest}

The authors declare no conflict of interest.

Acknowledgements. We would like to thank May Yu for assistance with cell culture. YAl is supported by the Sir Fredrick Banting and Dr. Charles Best Canada Graduate Scholarship provided by the Canadian Institutes of Health Research (ClHR). This research was funded by ClHR grant MOP119605.

1. Rudnicka AR, Mt-Isa S, Owen CG, Cook DG, Ashby D. Variations in primary open-angle glaucoma prevalence by age, gender, and race: a Bayesian meta-analysis. Invest Ophthalmol Vis Sci 2006; 10: 4254-4261. 
2. Leske MC. Open-angle glaucoma - an epidemiologic overview. Ophthalmic Epidemiol 2007; 14: 166-172.

3. Shields MB. Axenfeld-Rieger syndrome: a theory of mechanism and distinctions from the iridocorneal endothelial syndrome. Trans Am Ophthalmol Soc 1983; 81: 736-784.

4. Mears AJ, Mirzayans F, Gould DB, Pearce WG, Walter MA. Autosomal dominant iridogoniodysgenesis anomaly maps to 6p25. Am J Hum Genet 1996; 59: 1321-1327.

5. Mears AJ, Jordan T, Mirzayans F, Dubois S, Kume T, Parlee M et al. Mutations of the forkhead/winged-helix gene, FKHL7, in patients with Axenfeld-Rieger anomaly. Am J Hum Genet 1998; 63: 1316-1328.

6. Nishimura DY, Swiderski RE, Alward WL, Searby CC, Patil SR, Bennet SR et al. The forkhead transcription factor gene FKHL7 is responsible for glaucoma phenotypes which map to 6p25. Nat Genet 1998; 19: 140-147.

7. Tumer Z, Bach-Holm D. Axenfeld-Rieger syndrome and spectrum of PITX2 and FOXC1 mutations. Eur J Hum Genet 2009; 17: 1527-1539.

8. Saleem RA, Banerjee-Basu S, Berry FB, Baxevanis AD, Walter MA. Analyses of the effects that disease-causing missense mutations have on the structure and function of the wingedhelix protein FOXC1. Am J Hum Genet 2001; 68: 627-641.

9. Ito YA, Footz TK, Murphy TC, Courtens W, Walter MA. Analyses of a novel L130F missense mutation in FOXC1. Arch Ophthalmol 2007; 125: 128-135.

10. Ito YA, Footz TK, Berry FB, Mirzayans F, Yu M, Khan AO et al. Severe molecular defects of a novel FOXC1 W152G mutation result in aniridia. Invest Ophthalmol Vis Sci 2009; 50 3573-3579.

11. Berry FB, Skarie JM, Mirzayans F, Fortin Y, Hudson TJ, Raymond V et al. FOXC1 is required for cell viability and resistance to oxidative stress in the eye through the transcriptional regulation of FOXO1A. Hum Mol Genet 2008; 17: 490-505.

12. Wang WH, McNatt LG, Shepard AR, Jacobson N, Nishimura DY, Stone EM et al. Optimal procedure for extracting RNA from human ocular tissues and expression profiling of the congenital glaucoma gene FOXC1 using quantitative RT-PCR. Mol Vis 2001; 7: 89-94.

13. Rose RC, Richer SP, Bode AM. Ocular oxidants and antioxidant protection. Proc Soc Exp Biol Med 1998; 217: 397-407.

14. Ramachandran S, Morris SM, Devamanoharan P, Henein M, Varma SD. Radio-isotopic determination of hydrogen peroxide in aqueous humor and urine. Exp Eye Res 1991; 53: 503-506.

15. Valko M, Leibfritz D, Moncol J, Cronin MT, Mazur M, Telser J. Free radicals and antioxidants in normal physiological functions and human disease. Int J Biochem Cell Biol 2007; 39: 44-84.

16. Kumar DM, Agarwal N. Oxidative stress in glaucoma: a burden of evidence. J Glaucoma 2007; 16: 334-343.

17. Caballero M, Liton PB, Challa P, Epstein DL, Gonzalez P. Effects of donor age on proteasome activity and senescence in trabecular meshwork cells. Biochem Biophys Res Commun 2004; 323: 1048-1054.

18. de Nadal E, Ammerer G, Posas F. Controlling gene expression in response to stress Nat Rev Genet 2011; 12: 833-845.

19. Sorkhabi R, Ghorbanihaghjo A, Javadzadeh A, Rashtchizadeh N, Moharrery M. Oxidative DNA damage and total antioxidant status in glaucoma patients. Mol Vis 2011; 17: 41-46.

20. Vittitow J, Borras T. Genes expressed in the human trabecular meshwork during pressureinduced homeostatic response. J Cell Physiol 2004; 201: 126-137.

21. Bradley JM, Kelley MJ, Rose A, Acott TS. Signaling pathways used in trabecular matrix metalloproteinase response to mechanical stretch. Invest Ophthalmol Vis Sci 2003; 44: 5174-5181.

22. Leung TK, Rajendran MY, Monfries C, Hall C, Lim L. The human heat-shock protein family. Expression of a novel heat-inducible HSP70 (HSP70B') and isolation of its CDNA and genomic DNA. Biochem J 1990; 267: 125-132.

23. Noonan E, Giardina C, Hightower L. Hsp70B' and Hsp72 form a complex in stressed human colon cells and each contributes to cytoprotection. Exp Cell Res 2008; 314: 2468-2476.

24. Noonan EJ, Fournier G, Hightower LE. Surface expression of Hsp70B' in response to proteasome inhibition in human colon cells. Cell Stress Chaperones 2008; 13: 105-110.

25. Noonan EJ, Place RF, Rasoulpour RJ, Giardina C, Hightower LE. Cell number-dependent regulation of Hsp70B' expression: evidence of an extracellular regulator. J Cell Physiol 2007; 210: 201-211.
26. Beere HM, Wolf BB, Cain K, Mosser DD, Mahboubi A, Kuwana T et al. Heat-shock protein 70 inhibits apoptosis by preventing recruitment of procaspase- 9 to the Apaf-1 apoptosome. Nat Cell Biol 2000; 2: 469-475.

27. Kim HE, Jiang X, Du F, Wang X. PHAPI, CAS, and Hsp70 promote apoptosome formation by preventing Apaf-1 aggregation and enhancing nucleotide exchange on Apaf-1. Mol Cell 2008; 30: 239-247.

28. Saleh A, Srinivasula SM, Balkir L, Robbins PD, Alnemri ES. Negative regulation of the Apaf-1 apoptosome by Hsp70. Nat Cell Biol 2000; 2: 476-483.

29. Ravagnan L, Gurbuxani S, Susin SA, Maisse C, Daugas E, Zamzami N et al. Heat-shock protein 70 antagonizes apoptosis-inducing factor. Nat Cell Biol 2001; 3: 839-843.

30. Stern S, Dror T, Stolovicki E, Brenner N, Braun E. Genome-wide transcriptional plasticity underlies cellular adaptation to novel challenge. Mol Syst Biol 2007; 3: 106.

31. Kampinga HH, Craig EA. The HSP70 chaperone machinery: J proteins as drivers of functional specificity. Nat Rev Mol Cell Biol 2010; 11: 579-592.

32. Akerfelt M, Morimoto RI, Sistonen L. Heat shock factors: integrators of cell stress, development and lifespan. Nat Rev Mol Cell Biol 2010; 11: 545-555.

33. Gallucci S, Matzinger P. Danger signals: SOS to the immune system. Curr Opin Immuno 2001; 13: 114-119.

34. Saito K, Dai Y, Ohtsuka K. Enhanced expression of heat shock proteins in gradually dying cells and their release from necrotically dead cells. Exp Cell Res 2005; 310: 229-236.

35. Calderwood SK, Mambula SS, Gray PJ Jr. Extracellular heat shock proteins in cell signaling and immunity. Ann N Y Acad Sci 2007; 1113: 28-39.

36. Calnan DR, Brunet A. The FoxO code. Oncogene 2008; 27: 2276-2288.

37. Berry FB, Mirzayans F, Walter MA. Regulation of FOXC1 stability and transcriptional activity by an epidermal growth factor-activated mitogen-activated protein kinase signaling cascade. J Biol Chem 2006; 281: 10098-10104.

38. Lehmann OJ, Ebenezer ND, Jordan T, Fox M, Ocaka L, Payne A et al Chromosomal duplication involving the forkhead transcription factor gene FOXC1 causes iris hypoplasia and glaucoma. Am J Hum Genet 2000; 67: 1129-1135.

39. Lehmann OJ, Ebenezer ND, Ekong R, Ocaka L, Mungall AJ, Fraser $S$ et al. Ocular developmental abnormalities and glaucoma associated with interstitial 6p25 duplications and deletions. Invest Ophthalmol Vis Sci 2002; 43: 1843-1849.

40. Nishimura DY, Searby CC, Alward WL, Walton D, Craig JE, Mackey DA et al. A spectrum of FOXC1 mutations suggests gene dosage as a mechanism for developmental defects of the anterior chamber of the eye. Am J Hum Genet 2001; 68: 364-372.

41. Ray PS, Wang J, Qu Y, Sim MS, Shamonki J, Bagaria SP et al. FOXC1 is a potential prognostic biomarker with functional significance in basal-like breast cancer. Cancer Res 2010; 70: 3870-3876.

42. Tonoki H, Harada N, Shimokawa O, Yosozumi A, Monzaki K, Satoh K et al. AxenfeldRieger anomaly and Axenfeld-Rieger syndrome: clinical, molecular-cytogenetic, and DNA array analyses of three patients with chromosomal defects at 6p25. Am J Med Genet 2011; 155A: 2925-2932.

43. Schwechter BR, Millet LE, Levin LA. Histone deacetylase inhibition-mediated differentiation of RGC-5 cells and interaction with survival. Invest Ophthalmol Vis Sci 2007; 48: 2845-2857

44. Kozlowski K Walter MA. Variation in residual PITX2 activity underlies the phenotypic spectrum of anterior segment developmental disorders. Hum Mol Genet 2000; 9: 2131-2139

45. Huang L, Chi J, Berry FB, Footz TK, Sharp MW, Walter MA. Human p32 is a novel FOXC1 interacting protein that regulates FOXC1 transcriptional activity in ocular cells. Invest Ophthalmol Vis Sci 2008; 49: 5243-5249.

46. Tamimi Y, Skarie JM, Footz T, Berry FB, Link BA, Walter MA. FGF19 is a target for FOXC1 regulation in ciliary body-derived cells. Hum Mol Genet 2006; 15: 3229-3240.

(c) $(9)$ Cell Death and Disease is an open-access journal cublished by Nature Publishing Group. This work is licensed under a Creative Commons Attribution-NonCommercialNoDerivs 3.0 Unported License. To view a copy of this license, visit http://creativecommons.org/licenses/by-nc-nd/3.0/

Supplementary Information accompanies this paper on Cell Death and Disease website (http://www.nature.com/cddis) 